\title{
Transcriptomic study of the mechanism of anoikis resistance in head and neck squamous carcinoma
}

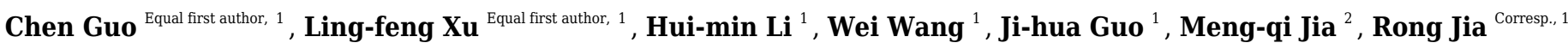 \\ , Jun Jia ${ }^{\text {Corresp. } 1,2}$ \\ ${ }^{1}$ The State Key Laboratory Breeding Base of Basic Science of Stomatology (Hubei-MOST) \& Key Laboratory of Oral Biomedicine Ministry of Education, \\ Wuhan University, School and Hospital of Stomatology, Wuhan, Hubei, China \\ 2 Department of Oral and Maxillofacial Surgery, Wuhan University, School and Hospital of Stomatology, Wuhan, Hubei, China \\ Corresponding Authors: Rong Jia, Jun Jia \\ Email address: jiarong@whu.edu.cn, junjia@whu.edu.cn
}

Background: Normal epithelial cells rapidly undergo apoptosis as soon as they lose contact with the extracellular matrix (ECM), which is termed as anoikis. However, cancer cells tend to develop a resistance mechanism to anoikis. This acquired ability is termed as anoikis resistance. Cancer cells, with anoikis resistance, can spread to distant tissues or organs via the peripheral circulatory system and cause cancer metastasis. Thus, inhibition of anoikis resistance blocks the metastatic ability of cancer cells.

Methods: Anoikis-resistant CAL27 (CAL2 $7^{A R}$ ) cells were induced from CAL27 cells using the suspension culture approach. Transcriptome analysis was performed using RNA-Seq to study the differentially expressed genes (DEGs) between the CAL27 $7^{A R}$ cells and the parental CAL27 cells. Gene function annotation and Gene Ontology (GO) enrichment analysis were performed using DAVID database. Signaling pathways involved in DEGs were analyzed using Gene Set Enrichment Analysis (GSEA) software. Analysis results were confirmed by reverse transcription PCR (RT-PCR), Western blotting, and gene correlation analysis based on the TCGA database.

Results: GO enrichment analysis indicated that the biological process (BP) of the DEGs was associated with epidermal development, DNA replication, and G1/S transition of the mitotic cell cycle. The analysis of cellular component (CC) showed that the most significant up-regulated genes were related to extracellular exosome. KEGG Pathway analysis revealed that 23 signaling pathways were activated ( $p$ value $\leq 0.05$, FDR $q$-value $\leq 0.05$ ) and 22 signaling pathways were suppressed ( $p$-value $\leq 0.05$, FDR $q$ value $\leq 0.05)$. The results from the GSEA indicated that in contrast to the inhibition of EGFR signaling pathway, the VEGF signaling pathway was activated. The VEGF signaling pathway possibly activates STAT3 though induction of STAT3 phosphorylation. Gene correlation analysis revealed that the VEGFASTAT3-KLF4-CDKN1A signal axis was not only present in head and neck squamous carcinoma (HNSCC) but also other two epithelial-derived carcinomas that highly express VEGFA, including kidney renal clear cell carcinoma (KIRC) and ovarian serous cystadenocarcinoma (OV). 


\section{Transcriptomic study of the mechanism of anoikis resistance \\ 2 in head and neck squamous carcinoma}

3 Chen Guo ${ }^{1} \cdot$ Ling-feng Xu${ }^{1} \cdot$ Hui-min $\mathrm{Li}^{1} \cdot$ Wei Wang ${ }^{1} \cdot$ Ji-hua Guo ${ }^{1} \cdot$ Meng-qi Jia ${ }^{2} \cdot$ Rong Jia $^{1} \cdot \mathrm{Jun} \mathrm{Jia}^{1,2}$

$4 \quad{ }^{1}$ The State Key Laboratory Breeding Base of Basic Science of Stomatology (Hubei-MOST) \& Key Laboratory

5 of Oral Biomedicine Ministry of Education, School and Hospital of Stomatology, Wuhan University, Wuhan

6 430079, China

$7 \quad 2$ Department of Oral and Maxillofacial Surgery, School and Hospital of Stomatology, Wuhan University,

8 No237, Luoyu Road, Hongshan District, Wuhan 430079, China.

9 Chen Guo and Ling-feng Xu contributed equally to this work.

10 Corresponding authors:

11 Rong Jia and Jun Jia

12 No237, Luoyu Road, Hongshan District, Wuhan 430079, China.

13 Email address: junjia@whu.edu.cn; jiarong@whu.edu.cn 


\section{Abstract:}

16 Background: Normal epithelial cells rapidly undergo apoptosis as soon as they lose contact with the

17 extracellular matrix (ECM), which is termed as anoikis. However, cancer cells tend to develop a resistance

18 mechanism to anoikis. This acquired ability is termed as anoikis resistance. Cancer cells, with anoikis resistance,

19 can spread to distant tissues or organs via the peripheral circulatory system and cause cancer metastasis. Thus,

20 inhibition of anoikis resistance blocks the metastatic ability of cancer cells.

21 Methods: Anoikis-resistant CAL27 (CAL27 ${ }^{\mathrm{AR}}$ ) cells were induced from CAL27 cells using the suspension

22 culture approach. Transcriptome analysis was performed using RNA-Seq to study the differentially expressed

23 genes (DEGs) between the CAL27 ${ }^{\mathrm{AR}}$ cells and the parental CAL27 cells. Gene function annotation and Gene

24 Ontology (GO) enrichment analysis were performed using DAVID database. Signaling pathways involved in

25 DEGs were analyzed using Gene Set Enrichment Analysis (GSEA) software. Analysis results were confirmed

26 by reverse transcription PCR (RT-PCR), Western blotting, and gene correlation analysis based on the TCGA

27 database.

28 Results: GO enrichment analysis indicated that the biological process (BP) of the DEGs was associated with

29 epidermal development, DNA replication, and G1/S transition of the mitotic cell cycle. The analysis of cellular

30 component (CC) showed that the most significant up-regulated genes were related to extracellular exosome.

31 KEGG Pathway analysis revealed that 23 signaling pathways were activated (p-value $\leq 0.05$, FDR q-value $\leq$

320.05 ) and 22 signaling pathways were suppressed ( $p$-value $\leq 0.05$, FDR q-value $\leq 0.05$ ). The results from the

33 GSEA indicated that in contrast to the inhibition of EGFR signaling pathway, the VEGF signaling pathway was 
34 activated. The VEGF signaling pathway possibly activates STAT3 through induction of STAT3

35 phosphorylation. Gene correlation analysis revealed that the VEGFA-STAT3-KLF4-CDKN1A signal axis was

36 not only present in head and neck squamous carcinoma (HNSCC) but also other two epithelial-derived

37 carcinomas that highly express VEGFA, including kidney renal clear cell carcinoma (KIRC) and ovarian serous

38 cystadenocarcinoma $(\mathrm{OV})$.

39 


\section{Introduction}

41 In 2012, 529,500 patients suffered from lip, oral cavity, and pharyngeal cancers globally, accounting for 3.8\%

42 of all cancer cases. It is predicted that by 2035 , the incidence of lip, oral and pharyngeal cancer will increase by

$4362 \%$, reaching to 856000 cases (Shield et al. 2017). Head and neck squamous cell carcinoma (HNSCC), which

44 is mainly consisted of cancers from lip, oral cavity, and pharynx, accounts for $90 \%$ of head and neck cancers

45 (Suh et al. 2014). Noticeably, there were several reports that claimed that the long-term prognosis of many

46 patients with HNSCC is poor due to cancer distant metastasis (Li et al. 2017; Wreesmann et al. 2004). Cancer

47 distant metastasis includes four main steps: invasion, intravasation, dissemination and colonization ( $\mathrm{Su}$ et al.

48 2015). The prerequisite of dissemination is that cancer cells could acquire the ability to resist anoikis and survive

49 in the lymphatic and circulatory system (Yan et al. 2005). Anoikis is a kind of cell programmed death which

50 occurs as a result of cell detachment from the extracellular matrix (ECM). For this reason, metastasis is

51 inefficient when cancer cells undergo anoikis (Kim et al. 2012b). Unfortunately, a few cancer cells are likely to

52 develop the ability to resist anoikis, which helps them to survive in the circulatory system (Simpson et al. 2008).

53 There are several mechanisms that endow cancer cells with the characteristics of anoikis resistance, including

54 the following: (1) the activation of the anti-apoptotic signaling pathways, such as integrin signaling pathway and

$55 \mathrm{PI} 3 \mathrm{~K} /$ Akt signaling pathway, which allows transformed cancer cells to survive in the circulatory system (Attwell

56 et al. 2000; Davies et al. 1999; Vitolo et al. 2009); (2) secretion of autocrine growth factors, such as EDIL3 and

57 VEGFA, which aids proliferation, survival and migration (Feng et al. 2014; Sher et al. 2009); and (3)

58 overexpression of Twist, HGF/Met and NF-kB through epithelial mesenchymal transformation (EMT) 
59 (Thompson et al. 2005). Research relating to the mechanisms contributing to anoikis resistance can provide us

60 with an opportunity to limit anoikis resistance, which would prevent cancer cell metastasis.

61 Many studies aimed to reveal the mechanism of anoikis resistance in HNSCC (Liao et al. 2017; Shen et al. 2017;

62 Yadav et al. 2011; Zeng et al. 2002). EMT phenomenon is widely accepted as one the most important factors for

63 cancer cells acquiring anoikis resistance (Taddei et al. 2012). Transcriptional profiling has identified that the

64 strongly upregulated transcripts include genes that are reportedly involved in invasion and metastasis, such as

65 Tbx3, DOCK10, LOX, ROBO1 and SRGN, following induction of EMT in HNSCC (Humtsoe et al. 2012).

66 Moreover, Yan demonstrated that NF- $\mathrm{BB}$, as a transcriptional regulator of many genes, is associated with

67 inflammatory mediator production and apoptosis. They put forward that detachment could directly activates NF-

$68 \mathrm{\kappa B}$ and that the NF- $\mathrm{kB}$ activation has positive effect on the early course of anoikis because of inflammation

69 factor stimulation generated by NF-kB activation (Yan et al. 2005). Which genes play significant roles in anoikis

70 resistance in HNSCC remains unknown. In this study, we conducted transcriptional studies on anoikis resistance

71 in HNSCC. Anoikis-resistant CAL2 $7^{\mathrm{AR}}$ cells were acquired using a suspension culture approach (Kupferman et

72 al. 2007), and then RNA-Seq was used to obtain deep sequencing data for whole transcriptome analysis between

73 CAL27 cells and CAL27 ${ }^{\mathrm{AR}}$ cells, which helped us to obtain a large number of differentially expressed genes

74 (DEGs) between these two kinds of cells. Through bioinformatics analysis and verification of DEGs, we found

75 several signaling pathways related to the ability of anoikis resistance in CAL27 ${ }^{A R}$ cells. Furthermore, we

76 analyzed these signaling pathways and found a signal axis related to the ability of anoikis resistance in multiple 
77 epithelial tumors that overexpressed VEGFA. Our study, for the first time, reveals the changes in transcriptional

78 level of anoikis-resistant head and neck squamous cell carcinoma cells. 


\section{Materials and methods}

\section{CAL27 cells monolayer culture}

82 Oral squamous cell carcinoma cell line, CAL27, was maintained in adherent culture conditions and was

83 cultivated in Dulbecco's Modified Eagle Medium (DMEM, Hyclone, South Logan, UT, USA) supplemented

84 with $10 \%$ fetal bovine serum (FBS, Hyclone) and $1 \%$ antibiotic-antimycotic solution in an incubator with moist

85 air containing $5 \% \mathrm{CO}_{2}$ at $37^{\circ} \mathrm{C}$.

\section{Collection for CAL27 cells resisting to anoikis}

87 To collect anoikis-resistant CAL27 cells, according to previously reported procedures by Kupferman et al.

88 (Kupferman et al. 2007), we digested approximately $2 \times 10^{8}$ CAL27 cells using $0.25 \%$ trypsin with $0.1 \%$ EDTA

89 solution from a monolayer grown in a tissue culture flask. Then, the CAL27 cells were transferred to a $10 \%$

90 poly-HEMA (Sigma Chemical Co. USA) coated tissue culture dish $(10 \mathrm{~cm})$ for $72 \mathrm{~h}$ in an incubator with moist

91 air containing $5 \% \mathrm{CO}_{2}$ at $37{ }^{\circ} \mathrm{C}$. Subsequently, these CAL27 spheroids cells were centrifuged at $300 \mathrm{rpm}$ for 5

$92 \mathrm{~min}$. Then, they were transferred to an uncoated dish, and were allowed to replicate for $24 \mathrm{~h}$. After six rounds of

93 coated and un-coated culture, anoikis-resistant CAL27 cells were harvested at $300 \mathrm{rpm}$ for 5 min; they were

94 marked as CAL27 $7^{\mathrm{AR}}$ cells.

95 RNA-seq and data analysis

96 Total RNA was isolated from the three CAL27 samples and three CAL27 ${ }^{\mathrm{AR}}$ samples using TRIzol (Invitrogen,

97 USA) according to the manufacturer's instructions. The concentration and quality of RNA samples were 
OmicShare tools (http://www.omicshare.com/tools/). The raw data have been deposited at the National Center

114 for Biotechnology Information Sequence Read Archive (SRA) with associated accession numbers SRP158985. 115 The workflow of data analysis was summarized in Fig. S1.

determined using a Nano Drop 2000 micro-volume spectrophotometer (Thermo Scientific, USA). Library construction and sequencing were performed by the BGI Genomics Institute, Wuhan, China (https://www.bgi.com/). The library preparation was followed by BGI's standard procedure. The libraries were sequenced on the Illumina HiSeq 2000 platform using the 50-bp pair-end sequencing strategy and 60 million reads were generated for each sample. DEGs screening aimed to find DEGs among samples and perform further function analysis on them. We used Noiseq package method for screening analysis of DEGs. First, Noiseq use sample's gene expression in each group to calculate $\log 2$ (foldchange) $\mathrm{M}$ and absolute different value $\mathrm{D}$ of all pair conditions to build noise distribution model. Second, for gene A, Noiseq computes its average expression "Control-avg" in control group and average expression "Treat-avg" in treatment group. Then the foldchange $(\mathrm{MA}=\log 2(($ Control-avg $) /($ Treat-avg $)))$ and absolute different value $\mathrm{D}(\mathrm{DA}=\mid$ Congrol-avg-Treat-avg $\mid)$ will be got. If MA and DA diverge from noise distribution model markedly, gene A will be defined as a DEG. "Loget" represents $\log 2$ fold change(Pacholewska 2017). "Probability" represents probability of differential expression. Noiseq obtains probability to assess the reliability of the difference(Tarazona et al. 2011). In order to visualize the distribution of DEGs among samples, cluster analysis of differentially expressed genes was carried out. We chose the top DEGs ( $\mid$ Loget $\mid \geq 3$, probability $\geq 0.9$ ) for display. Heatmapping of top DEGs was generated by

16 Database for Annotation, Visualization and Integrated Discovery (DAVID) functional annotation analysis 
117 To perform gene ontology (GO) enrichment analysis, DEGs (|Loget $\mid \geq 2$, probability $\geq 0.9$ ) were explored with

118 DAVID functional annotation tool (http://david.abcc.ncifcrf.gov) (Huang et al. 2008; Huang et al. 2009). Among

119 the enrichment results of up- or down-regulated DEGs, the top ten statistically significant enriched terms (p-

120 value $\leq 0.05$ and FDR $\leq 0.05$ ) of biological processes, molecular function and cellular component, as well as

121 three ontologies of GO, were picked up separately. Regarding the visualization of GO enrichment analysis

122 results, the top ten statistically significant enriched terms were visualized using an online tool

123 (http://www.ehbio.com). We further used DAVID to re-analyze the genes enriched in the terms of "protein

124 binding" and up- or down-regulated genes enriched in the "VEGFA_UP. V1_UP" and "VEGFA_UP. V1_DN"

125 terms. $p$-value $\leq 0.05$ and FDR $\leq 0.05$ was considered as statistically significant.

\section{Gene Set Enrichment Analysis (GSEA)}

127 Activated or repressive pathways were detected using Gene Set Enrichment Analysis (GSEA) (GSEA Desktop v3.0, Broad Institute) (www.broadinstitute.org/gsea/index.jsp) (Mootha et al. 2003; Subramanian et al. 2005).

Briefly, the "number of permutations" was set to 1000, the "collapse data set to gene symbols" was set to False, the "enrichment statistic" was set to Weighted, the "metric for ranking genes" was set to Singal2Noise, and the

131 "permutation type" was set to gene set. The Molecular Signatures Database (MSigDB) collected a large quantity

132 of annotated gene sets for analysis with the GSEA software. MSigDB gene sets are divided into 8 major

133 collections. C2 collection and C6 collection in the MSigDB were used to detect activated or repressive pathways

134 related to our DEGs, respectively. The used gene sets for enrichment analysis included "CP: KEGG: KEGG

135 gene sets" (Gene sets derived from the KEGG pathway database.), "CP: REACTOME: Reactome gene sets" 
136 (Gene sets derived from the Reactome pathway database) and "C6: oncogenic signatures" (Gene sets that

137 represent signatures of cellular pathways). $\mathrm{p}$-value $\leqslant 0.05$ and FDR q-value $\leqslant 0.05$ was considered as 138 statistically significant.

\section{Gene expression analysis}

140 VEGFA gene expression analysis in different types of tumors was done using GEPIA, an online tool for 141 analyzing the RNA sequencing expression data from TCGA (http://gepia.cancer-pku.cn/index.html) (Tang et al.

142 2017). The steps were as follows: The "Gene Expression Profile" tool was used; "VEGFA" was input into the

143 "Gene column"; "Differential Methods" was set to "ANOVA"; “|Log2FC| Cutoff” was set to "1"; "p-value

144 Cutoff" was set to "0.01"; "Log Scale" was set to "No"; "Matched Normal data" was set to "Match TCGA

145 normal and GTEx data". All types of cancer names, except mesothelioma and uveal melanoma, from the

146 "Dataset" was added to "Tissue Order". To better demonstrate the expression of VEGFA in tumor tissues and

147 normal tissues, we also created a box plot for the expression of VEGFA. Briefly, the "BoxPlot" tool was selected,

148 and "VEGFA" was inputted into the "Gene", "|Log2FC| Cutoff" was set to "1", "p-value Cutoff" was set to 149 "0.01", "Log Scale" was set to "YES", and HNSC, KIRC, OV and GBM were added into "Datasets".

\section{Gene correlation analysis}

151 Gene correlation analysis was done using GEPIA. The "correlation coefficient" was set to "Spearman". "HNSC

152 Tumor", "KIRC Tumor", "OV Tumor" and "GBM Tumor" were added into the "Used Expression Datasets"

153 column, respectively. $p$-value $\leqslant 0.05$ was considered as statistically significant. 


\section{Reverse transcription PCR (RT-RCR)}

155 Total cellular RNA was extracted using a Multisource Total RNA Miniprep Kit (Axygen, Tewksbury, MA,

156 USA). RNA was treated with DNase I (Invitrogen) and reverse-transcribed using random primers

157 (hexadeoxynucleotides) (Promega, Madison, WI, USA) and Moloney Murine Leukemia Virus (MMLV)

158 Reverse Transcriptase (Promega). PCR was performed using rTaq DNA polymerase (TaKaRa, Tokyo, Japan).

159 The primer sequences are listed in the Table S1.

160 Western blotting

161 Protein samples in the $2 \times$ SDS sample buffer were denatured by boiling for $5 \min$ in $95^{\circ} \mathrm{C}$, separated by $10 \%$

162 SDS-PAGE gels and transferred to a nitrocellulose membrane. The membrane was blotted with 5\% nonfat dry

163 milk for $1 \mathrm{~h}$ at room temperature and then probed with dilutions recommended by the primary antibody suppliers

164 overnight at $4^{\circ} \mathrm{C}$, including mouse monoclonal antibodies against STAT3 Tyr 705 (sc-8059, Santa Cruz, USA),

165 mouse monoclonal antibodies against STAT3 Ser 727 (sc-136193, Santa Cruz, USA) and mouse monoclonal

166 antibodies against GAPDH (sc-47724, Santa Cruz, USA).

167 


\section{Results}

\section{Cluster analysis of DEGs and RT-PCR confirmation}

170 To identify DEGs between CAL27 ${ }^{\mathrm{AR}}$ cells and CAL27 cells, we illustrated the gene expression features of

171 CAL27 $7^{\text {AR }}$ cells and CAL27 cells using RNA sequencing (RNA-seq) technology. The number of significantly

172 changed DEGs between CAL27 ${ }^{\mathrm{AR}}$ and CAL27cells was 2,847 ( $\mid$ Loget $\mid \geq 1$, probability $\geq 0.8$ ), of which 1,558

173 genes were up-regulated and 1,289 genes were down-regulated in CAL27 ${ }^{\text {AR }}$ cells (File S1). Furthermore, we

174 performed cluster analysis of the top 360 genes ( $\mid$ Loget $\mid \geq 3$, probability $\geq 0.9$ ) expression patterns. The

175 significantly up-regulated and down-regulated genes are shown in the cluster heatmap (Fig. 1A and Fig. S2).

176 The result of cluster analysis indicated that the samples had low heterogeneity and high repeatability. In addition,

177 we verified the results of RNA-Seq using RT-PCR. All the detected 21 genes, including 15 keratin-related genes

178 and 6 randomly selected other genes, matched the results of RNA-Seq, which confirmed that the high-throughput

179 sequencing results were reliable (Fig. 1B).

180 Functional annotation analysis of DEGs was done using Database for Annotation, Visualization and

181 Integrated Discovery (DAVID).

182 To facilitate the comprehensive analysis of DEGs, total of 487 up-regulated genes and 335 down-regulated genes

183 ( $\mid$ Loget $\mid \geq 2$, probability $\geq 0.9$ ) were integrated to gene ontology (GO) terms using DAVID. The GO classification

184 was divided into three categories: biological process (BP), molecular function (MF) and cellular component

185 (CC). In each category, top ten significant terms enriched in the up- or down-regulated genes were shown in the

186 Figure 2. Regarding biological process, many up-regulated genes were associated with "epidermis development" 
$187\left(\mathrm{p}\right.$-value $\left.=1.01 \times 10^{-21}\right)$, "keratinocyte differentiation" $\left(\mathrm{p}\right.$-value $\left.=1.62 \times 10^{-20}\right)$ and "keratinization" $(\mathrm{p}$-value $=$

$\left.1881.65 \times 10^{-14}\right)$. In contrast, the top three terms in down-regulated genes were "cell division" $\left(p-v a l u e=4.49 \times 10^{-33}\right)$,

189 "mitotic nuclear division" (p-value $\left.=6.38 \times 10^{-27}\right)$ and "DNA replication" (p-value $\left.=1.24 \times 10^{-21}\right)(F i g .2 A)$. The

190 molecular functions of up- or down-regulated genes were closely related to "structural molecule activity" (p-

191 value $\left.=2.31 \times 10^{-9}\right)$ and "protein binding" $\left(\mathrm{p}\right.$-value $\left.=2.63 \times 10^{-12}\right)$ respectively. Moreover, we noticed that a great

192 number of up-regulated genes were enriched into "protein heterodimerization activity" $\left(p-v a l u e=4.62 \times 10^{-6}\right)$

193 and "calcium ion binding" (p-value $\left.=4.12 \times 10^{-5}\right)($ Fig. 2B). Most significantly down-regulated genes were

194 associated with protein binding, however, their biological functions were unclear. For this reason, we further re-

195 analyzed these genes related to protein binding using DAVID. The results revealed that most down-regulated

196 genes related to protein binding could influence "cell cycle" ( $p$-value $\left.=6.27 \times 10^{-21}\right)$, "DNA replication" ( $p$-value

$\left.197=3.33 \times 10^{-7}\right)$ and "p53 signaling pathway" ( $\mathrm{p}$-value $\left.=2.31 \times 10^{-4}\right)$. Interestingly, the results of cellular component

198 indicated that 146 up-regulated genes, including S100A8, S100A9, WNT4 and CD14, were enriched in the

199 "extracellular exosome" term $\left(\mathrm{p}\right.$-value $\left.=1.02 \times 10^{-20}\right)$. These up-regulated genes related to the "extracellular

200 exosome" term were summarized in Supplement Table 2. The results also indicated that 117 down-regulated

201 genes were enriched in the "nucleoplasm" term ( $\mathrm{p}$-value $\left.=7.76 \times 10^{-22}\right)$. Besides these positions, many up-

202 regulated gene products were enriched in the extracellular space $\left(\mathrm{p}\right.$-value $\left.=1.21 \times 10^{-10}\right)$ and extracellular region

$203\left(\mathrm{p}\right.$-value $\left.=9.22 \times 10^{-7}\right)$. Furthermore, there were a great number of down-regulated gene products located in the

204 nucleus $\left(\mathrm{p}-\mathrm{value}=1.10 \times 10^{-10}\right)($ Fig. 2 C $)$. All the results' FDR $\leq 0.05$. 
205 Gene Set Enrichment Analysis (GSEA) of DEGs revealed some important signaling pathways and characteristics of oncogene in CAL27 ${ }^{\mathrm{AR}}$ cells.

207 To further explore signal pathways of DEGs, the biological significance related with the DEGs was studied using

208 GSEA. Different collections of molecular signatures database (MSigDB) gene sets were used to detect biological significance of DEGs. These collections of MSigDB gene sets included chemical and genetic perturbations (CGP), KEGG gene sets, Reactome gene sets and oncogenic signatures. Activated signaling pathways included

211 drug metabolism cytochrome P450, PPAR signaling pathway, cell adhesion molecules (Cams), arachidonic

212 metabolism, linoleic metabolism and Toll like receptor signaling pathway (Fig. $3 A$ ). In addition, several

213 signaling pathways were suppressed in CAL27 ${ }^{\mathrm{AR}}$ cells, such as DNA replication, mismatch repair, nucleotide

214 excision repair and cell cycle pathways (Fig. 3B). The GSEA of Reactome pathways also indicated that some

215 cell cycle processes and events were suppressed. These cell cycle processes and events included G1/S specific

216 transcription, G0 and early G1, mitotic M/G1 phases, and G1/S transition (Table S3). Furthermore, we found

217 that "VEGF_A_UP.V1_UP" and "P53_DN.V2_UP" gene sets could be enriched in up-regulated genes, and

218 "VEGF_A_UP.V1_DN" gene sets could be enriched in down-regulated genes when the gene sets of oncogenic

219 signatures were used to analyze DEGs (Fig. 4A-C). The GSEA of CGP disclosed that the

“KOBAYASHI_EGFR_SIGNALING_24HR_UP" and "YAN_ESCAPE_FROM_ANOIKIS" gene sets could

221 be enriched in up-regulated genes, and the "KOBAYASHI_EGFR_SIGNALING_24HR_DN" gene set could be 222 enriched in down-regulated genes (Fig. 4D-F).

\section{Bypass signaling in EGFR pathway may influence the cell cycle}


224 Some up- or down-regulated genes were enriched in the terms "KOBAYASHI_EGFR_SIGNALING_24HR

Viloriapetit et al. 2001). Moreover, some studies have revealed that IL-6 and HGF could help cancer cells to acquire the ability of anoikis resistance (Fofaria \& Srivastava 2014; Zeng et al. 2002), suggesting bypass 
242 results suggested that the activation of the VEGFA signaling pathway blocked the cell cycle progression in 243 CAL27 $7^{\text {AR }}$ cells.

244 VEGFA-STAT3-KLF4-CDKN1A signal axis presents in multiple epithelial tumors with high expression 245 of VEGFA

246 A recent study reported by Zhao et al. demonstrated that VEGFA could promote phosphorylation of STAT3 and

247 increase KLF4 expression (Zhao et al. 2015). Thus, we then detected the expression of phospho-STAT3 (Tyr

248 705) and phospho-STAT3 (Ser 727) protein in CAL27 ${ }^{\text {AR }}$ cells using western blotting. Our results showed that

249 both CAL27 ${ }^{\text {AR }}$ and CAL27 cells could express phospho-STAT3 (Ser 727) protein. However, the expression of

250 phospho-STAT3 (Tyr 705) protein could only be detected in CAL27AR cells (Fig. 5C). Hall et al. reported that

251 transcription of Krüppel-like transcription factor 4 (KLF4) could be activated by phospho-STAT3 (Tyr 705)

252 (Hall et al. 2009). KLF4 could block cell cycle progression via up-regulating CDKN1A expression and

253 repressing TP53 transcription, which produces an anti-apoptotic effect (Rowland \& Peeper 2006). More

254 importantly, the cell cycle arrest mediated by KLF4 might be the outcome of inhibition of G1/S transition, as

255 KLF4 could activate CDKN1A transcription (Chen et al. 2001; Wei et al. 2006). For this reason, we detected

256 KLF4, TP53 and CDKN1A expression in CAL27 ${ }^{\mathrm{AR}}$ cells. Our results showed that the CDKN1A and KLF4

257 expression was up-regulated in CAL27 ${ }^{\mathrm{AR}}$ cells (Fig. 5B), which is in accordance with the results of RNA-Seq.

258 Although some reports showed that CDKN1A could induce G1/S transition arrest and that its expression is

259 regulated by TP53 (Cazzalini et al. 2003; El-Deiry et al. 1994; Riley et al. 2008), TP53 expression was down-

260 regulated in CAL27 ${ }^{\mathrm{AR}}$ cells (Fig. 5B). In addition, our enrichment results indicated that the p53 signaling

Peer] reviewing PDF | (2018:11:32868:2:0:NEW 9 Apr 2019) 
261 pathway was suppressed in CAL27 ${ }^{\mathrm{AR}}$ cells as some up-regulated genes were enriched in the term

262 "P53_DN.V2_UP" (Fig. 4B)- a gene set related to TP53 silence (Elkon et al. 2005). Consequently, the

263 overexpression of CDKN1A might be induced by KLF4 in CAL27 ${ }^{\text {AR }}$ cells rather than TP53. Thus, it is possible

264 that VEGFA-STAT3-KLF4-CDKN1 A signal axis might be present in CAL27 $7^{\mathrm{AR}}$ cells. To verify this view, based

265 on TCGA database, gene correlation analysis was used to detect correlations among VEGFA, STAT3, KLF4

266 and CDKN1A genes. The results of gene correlation analysis revealed that not only VEGFA expression was

267 positively related to KLF4 and CDKN1A expression in HNSCC (Fig. 5E, 5F), but also that STAT3 was

268 positively related to KLF4 and CDKN1A expression (Fig. 5G, 5H). In addition, the correlation between KLF4

269 and CDKN1A genes was also positive (Fig. 5I) in HNSCC. Overall, these results confirmed the fact that

270 VEGFA-STAT3-KLF4-CDKN1A signal axis might be present in CAL27 ${ }^{\text {AR }}$ cells.

271 To test whether the VEGFA-STAT3-KLF4-CDKN1A signal axis was accidental in HNSCC, we studied the

272 cancers with high expression of VEGFA based on TCGA database. Data from TCGA database indicated that

273 VEGFA is also expressed highly in kidney renal clear cell carcinomas (KIRC), ovarian serous

274 cystadenocarcinomas (OV) and glioblastoma (GBM), as well as HNSCC (Fig. 6A, 6B). What's more, we studied

275 the correlation among VEGFA, STAT3, KLF4 and CDKN1A genes in KIRC, OV and GBM, with the results

276 showing that the correlation among VEGFA, STAT3, KLF4 and CDKN1A genes in KIRC and OV was

277 consistent with that in HNSCC (Fig. 7A-J). This indicated that the VEGFA-STAT3-KLF4-CDKN1A signal axis

278 not only exists in the HNSCC, but also in the two other kinds of epithelial cancers that showed high expression

279 of VEGFA. However, the relationship between VEGFA and KLF4 genes have no statistical significance in 
280 glioblastoma (Fig. 7O). Therefore, whether the VEGFA-STAT3-KLF4-CDKN1A signal axis existed in 281 glioblastomas remains unclear. Thus far, the study has argued that VEGFA-STAT3-KLF4-CDKN1A signal axis 282 may play an important role in epithelial cancers with high expression of VEGFA, such as HNSCC, KIRC and 283 OV. 
285 Discussion

286 Exosome released by CAL27 ${ }^{\mathrm{AR}}$ cells might play a significant role in cancer metastasis

287 GO enrichment analysis revealed that the most significant up-regulated genes (Loget $\geq 2$, probability $\geq 0.9)$ are

related to cell differentiation, and the most significantly down-regulated genes (Loget $\leq-2$, probability $\geq 0.9$ )

are closely related to the cell cycle (Fig. 3A,3B). This indicated that active cell differentiation as well as relatively stable cell quiescence exist in CAL2 $7^{\mathrm{AR}}$ cells. This phenomenon is similar to the properties of cancer stem cells

(CSCs) (Clarke et al. 2006; Glauche et al. 2009). Chen et al. reported that cancer cells could be induced to generate a special cell group that have properties of CSCs through the suspension culture (Chen et al. 2012).

CSCs have a strong ability to secrete exosomes (Hannafon \& Ding 2015), which could explain why there are a large number of up-regulated genes associated with extracellular exosomes in CAL27 ${ }^{\mathrm{AR}}$ cells. These upregulated genes may contribute to the formation of tumor metastatic microenvironments. For example, Ji et al. demonstrated that S100A8 and S100A9 was overexpressed in exosomes, released by SW620 cells, an isogenic human colorectal cancer cell line (Ji et al. 2013). S100A8/A9 are regarded as promotors for cancer metastasis in multiple types of cancers, such as colorectal cancer, prostate cancer, gastric cancer and breast cancer (Hermani et al. 2006; Ji et al. 2013; Yin et al. 2013; Yong \& Moon 2007). In addition, a report has shown that the WNT signaling pathway has an essential role in maintaining stem and cancer stem cells (Holland et al. 2013).

301 Furthermore, Dimeo et al. stated that WNT signaling could drive tumor metastasis through mediating cancer cell

302 self-renewal (DiMeo et al. 2009). Moreover, Rebecca et al. demonstrated that WNT signaling is activated in 303 patient-derived metastatic cancer stem-like cells (Lamb et al. 2013). Importantly, there is evidence that Wnt4, 
304 derived from the exosomes of colorectal cancer (CRC) cells, could promote the migration of cancer cells and

305 angiogenesis by activating $\beta$-Catenin signaling (Lamb et al. 2013). Furthermore, Cheah et al. stated that CD14-

306 high bladder cancer (BC) cells could establish and maintain an immune suppressed, inflammatory tumor

307 microenvironment, which could be beneficial for bladder cancer metastasis. They demonstrated that CD14 is a

308 key gene with pro-tumorigenic effects in bladder cancer (Cheah et al. 2015). Overall, these cases demonstrated

309 that the up-regulated genes related to exosome might have a critical role in cancer metastasis in CAL27 ${ }^{\mathrm{AR}}$ cells

310 (Table S2).

311 Abnormal cellular metabolic pathways can activate the PPARs signaling pathway, thereby enhancing the

312 ability of anoikis resistance in $\mathrm{CAL27}{ }^{\mathrm{AR}}$ cells

313 Peroxisome proliferator-activated receptors (PPARs), a member of steroid receptor superfamily, are ligand

314 activated transcription factors (Issemann \& Green 1990). At present, there are three different subtypes of PPARs,

315 namely PPAR $\alpha, \operatorname{PPAR} \beta / \delta$ and PPAR $\gamma$ (Berger \& Moller 2002). These three subtypes are activated by

316 endogenous ligands and participate in the regulation of glucose, amino acid and lipid metabolism (Sertznig et al.

317 2007). At present, high expression of PPAR $\gamma$ was found in primary squamous cell carcinomas and squamous

318 cell carcinoma cell lines. Moreover, PPAR $\gamma$ could enhance the ability of anoikis resistance in squamous cell

319 carcinoma cells (Masuda et al. 2005). In addition, Shen et al. found that oleic acid (OA) promotes the expression

320 and autocrine regulation of angiopoietin like 4 (ANGPTL4) protein through the PPARs signaling pathway. High

321 expression of ANGPTL4 could also increase the anoikis resistance ability and promote cancer metastasis (Shen

322 et al. 2017). In this study, we also found that the PPARs signaling pathway is activated in CAL27 ${ }^{\text {AR }}$ cells (Fig.

Peer] reviewing PDF | (2018:11:32868:2:0:NEW 9 Apr 2019) 
323 3A). Moreover, the PPARs signaling pathway is accompanied with activation of abnormal cell metabolism

324 signaling pathways in CAL27 $7^{\mathrm{AR}}$ cells, such as arachidonic metabolism and linoleic metabolism signaling

325 pathways (Fig. 3A). It has been reported that the oxidized metabolites of arachidonic acid and linoleic acid could

326 be conducted with the PPAR signaling pathway activation (Chawla et al. 2001; Sheldrick et al. 2007;

327 Viswakarma et al. 2010). In addition, studies have shown that arachidonic acid could promote prostate cancer

328 cell growth (Vainio et al. 2011). It is also reported that linoleic acid could promote breast cancer cell migration

329 (Byon et al. 2009). Importantly, we also observed increased ANGPTL4 expression in CAL27 ${ }^{\text {AR }}$ cells (data not

330 shown). From this context, we inferred that abnormal cell metabolism was activated to adapt to the environment

331 of the suspension culture, which was supported by several reports (Christofk et al. 2008; Davison et al. 2013;

332 Schafer et al. 2009) These abnormal cell metabolisms could activate the PPARs signaling pathway. The

333 activation of PPARs signaling pathway could enhance the ability of anoikis resistance through increasing

334 ANGPTL4 expression in CAL27 ${ }^{\text {AR }}$ cells.

335 G1/S transition inhibition could provide a protection from anoikis for cancer cells with high expression of

336 VEGFA

337 Reginato et al. showed that the expression of EGFR was down-regulated after epithelial cells detachment from

338 ECM, as EGFR expression was dependent on the matrix signals (Reginato et al. 2003). We also found that EGFR

339 expression was lower in CAL27 ${ }^{\text {AR }}$ cells than CAL27 cells (Fig. 5A). In addition, results from GSEA of CGP

340 indicated that two gene sets related to EGFR inhibition were enriched in up- or down-regulated genes,

341 respectively (Fig. 4A, 4C). Thus, we inferred that the EGFR signaling pathway was suppressed in CAL27 AR 
342

343 (TKIs). However, the inhibition of the EGFR signaling pathway could be reversed by other signaling pathways,

344 such as VEGFA signaling pathway (Viloriapetit et al. 2001). Indeed, we found that VEGFA was up-regulated

cells. According to our knowledge, the EGFR signaling pathway could be inhibited by tyrosine kinase inhibitors in CAL2 $7^{\mathrm{AR}}$ cells (Fig. 5A). In addition, according to the results from the GSEA of oncogenic signatures, two gene sets related to VEGFA overexpression both were enriched in up- or down-regulated genes respectively (Fig. 4D, 4F). Thus, we supposed that the VEGFA signaling pathway might be activated in CAL27 ${ }^{\text {AR }}$ cells. So we inferred that the VEGFA signaling pathway, as a bypass signaling pathway in the EGFR signaling pathway, may play an important role in resisting the EGFR signaling pathway inhibition in CAL27 ${ }^{\mathrm{AR}}$ cells. Moreover, we found that many down-regulated genes followed by VEGFA overexpression could influence G1/S transition (Fig. 5D). Both GO analysis and GSEA analysis revealed that G1/S transition was suppressed because many down-regulated genes were significantly enriched in the "G1/S transition" term (Fig. 2A, Table S3), which suggested that $\mathrm{G} 1 / \mathrm{S}$ transition inhibition is a significant event in CAL27 ${ }^{\mathrm{AR}}$ cells.

Collins et al. stated that the G1/S transition inhibition could contribute to anoikis resistance in epithelial cells (Collins et al. 2005). We supported that G1/S transition inhibition might also be vital for anoikis resistance in CAL27 ${ }^{\mathrm{AR}}$ cells. As is known to us, CDKN1A (the gene that encodes p21) could block G1/S transition through inhibiting cyclin/Cdk complexes (Gartel \& Tyner 2002). We found that CDKN1A expression is up-regulated in CAL27 ${ }^{\text {AR }}$ cells (Fig. 5B). Gartel et al. stated that CDKN1A expression is divided into p53-dependent and p53independent mechanisms (Gartel \& Tyner 2002). As previously described, we found that overexpression of CDKN1A is a result of KLF4 activation rather than TP53 regulation in CAL27 ${ }^{\mathrm{AR}}$ cells. In this case, there is no 
361 doubt that both KLF4 and CDKN1A could help CAL27 ${ }^{\text {AR }}$ cells to acquire anoikis resistance through G1/S

362 transition inhibition. Moreover, we found that KLF4 transcription might be induced by phospho-STAT3 (Tyr

363 705) protein because phospho-STAT3 (Tyr 705) protein has been detected in CAL27 ${ }^{\text {AR }}$ cells. Several studies

364 revealed that STAT3 could enhance resistance to anoikis in pancreatic cancer cells, esophageal squamous cells

365 and melanoma cells (Du et al. 2009; Fofaria \& Srivastava 2014; Fofaria \& Srivastava 2015). In this case, the

366 STAT3-KLF4-CDKN1A signaling axis might be activated for enhancing anoikis resistance in CAL27 $7^{\mathrm{AR}}$ cells.

367 We also demonstrated that VEGFA might be an activator of STAT3 phosphorylation in CAL27 ${ }^{\text {AR }}$ cells. This

368 perspective was verified by gene correlation analysis that VEGFA expression was positively related to KLF4

369 and CDKN1A expression (Fig. 5E, 5F). Importantly, we demonstrated that VEGFA overexpression might lead

370 to G1/S transition inhibition (Fig. 5D). This result was in accordance with the effect of CDKN1A and KLF4

371 genes. Overall, cell cycle arrest at the G1 phase might prevent CAL27 ${ }^{\mathrm{AR}}$ cells from anoikis through activating

372 VEGFA-STAT3-KLF4-CDKN1A signaling axis. We have proven that VEGFA expression is up-regulated in

373 KIRC, OV and HNSCC tissues; furthermore, the VEGFA-STAT3-KLF4-CDKN1A signal axis also presents in

374 these three kinds of epithelial cancers tissues. Our study findings demonstrate that the VEGFA-STAT3-KLF4-

375 CDKN1A signaling axis might have a role in anoikis resistance in KIRC, OV and HNSCC.

376 Entry into quiescence driving by CDKN1A protected CAL27 ${ }^{\mathrm{AR}}$ cells from anoikis

377 The suppression of cell cycle signaling pathway in CAL27 ${ }^{\text {AR }}$ cells, which was confirmed by GSEA, is an

378 interesting phenomenon in the induction of anoikis resistance. Besides its role in cell metabolism, PPARs

379 signaling pathway could inhibit cell cycle through its function in transcriptional regulation (Altiok et al. 1997;

Peer] reviewing PDF | (2018:11:32868:2:0:NEW 9 Apr 2019) 
380 Miller et al. 2018; Toyota et al. 2002). Chung et al. put that activation of PPAR - $\gamma$ were able to induce cell cycle

381 arrest in anaplastic thyroid cancer cells via a p53 - independent, but CDKN1A dependent cytostatic pathway

382 (Chung et al. 2002). Interestingly, we found that the VEGFA-STAT3-KLF4-CDKN1A signaling axis was

383 activated during CAL27 ${ }^{\mathrm{AR}}$ cells detachment from ECM, which could result in the G1/S transition inhibition of

384 CAL27 $7^{\mathrm{AR}}$ cells. Moreover, CDKN1A has been demonstrated to be involved in the stemness acquirement of cancer stem cells (Kim \& Singh 2019), which could explain the plenty of exosomes excreted by CAL27 ${ }^{\text {AR }}$ cells.

Therefore, we propose that CDKN1A is a key regulator of anoikis resistance in CAL27 $7^{\mathrm{AR}}$ cells.

Perucca et al. put that high CDKN1A levels are indispensable for cells to enter and maintain the quiescence state

(Perucca et al. 2009). Quiescence is an actively maintained state rather than a passive state lacking proliferative activities, which could provide protection against cellular stress (Cheung \& Rando 2013; Coller et al. 2006; Sang resistance property in malignant pleural mesothelioma (Daubriac et al. 2009), which supports our proposal that cell quiescence induced by CDKN1A might protect CAL27 ${ }^{\mathrm{AR}}$ cells from anoikis. 


\section{Conclusions}

395 The acquirement of quiescence regulated by CDKN1A is critical for CAL27 ${ }^{\mathrm{AR}}$ cells survival from anoikis. In

396 addition, CAL27 ${ }^{\mathrm{AR}}$ cells may release several exosomes that have a pro-oncogenic role in HNSCC metastasis.

397 


\section{References}

399 Akhavan D, Pourzia AL, Nourian AA, Williams KJ, Nathanson D, Babic I, Villa GR, Tanaka K, Nael A, and

400

401

402

403

404

405

406

407

408

409

410

411

412

413

414

415

416

417

418

419

420

421

422

423

424

425

426

427

428

429

430

431

432

433

434

435
Yang H. 2013. De-repression of PDGFR $\beta$ transcription promotes acquired resistance to EGFR tyrosine kinase inhibitors in glioblastoma patients. Cancer discovery:CD-12-0502. 10.1158/21598290.CD-12-0502.

Altiok S, Xu M, and Spiegelman BM. 1997. PPAR $\gamma$ induces cell cycle withdrawal: inhibition of E2F/DP DNA-binding activity via down-regulation of PP2A. Genes \& development 11:1987-1998. 10.1101/gad.11.15.1987

Attwell S, Roskelley C, and Dedhar S. 2000. The integrin-linked kinase (ILK) suppresses anoikis. Oncogene 19:3811. 10.1038/sj.onc.1203711.

Berger J, and Moller DE. 2002. The mechanisms of action of PPARs. Annu Rev Med 53:409-435. 10.1146/annurev.med.53.082901.104018

Byon CH, Hardy RW, Ren C, Ponnazhagan S, Welch DR, McDonald JM, and Chen Y. 2009. Free fatty acids enhance breast cancer cell migration through plasminogen activator inhibitor-1 and SMAD4. Laboratory investigation 89:1221-1228. 10.1038/labinvest.2009.97.

Cazzalini O, Perucca P, Riva F, Stivala LA, Bianchi L, Vannini V, Ducommun B, and Prosperi E. 2003. p21CDKN1A does not interfere with loading of PCNA at DNA replication sites, but inhibits subsequent binding of DNA polymerase delta at the G1/S phase transition. Cell Cycle 2:596-603. 10.4161/cc.2.6.502.

Chawla A, Repa JJ, Evans RM, and Mangelsdorf DJ. 2001. Nuclear receptors and lipid physiology: opening the X-files. Science 294:1866-1870. 10.1126/science.294.5548.1866

Cheah MT, Chen JY, Sahoo D, Contreras-Trujillo H, Volkmer AK, Scheeren FA, Volkmer JP, and Weissman IL. 2015. CD14-expressing cancer cells establish the inflammatory and proliferative tumor microenvironment in bladder cancer. Proc Natl Acad Sci U S A 112:4725-4730. 10.1073/pnas.1424795112

Chen S-F, Chang Y-C, Nieh S, Liu C-L, Yang C-Y, and Lin Y-S. 2012. Nonadhesive culture system as a model of rapid sphere formation with cancer stem cell properties. PloS one 7:e31864. 10.1371/journal.pone.0031864.

Chen X, Johns DC, Geiman DE, Marban E, Dang DT, Hamlin G, Sun R, and Yang VW. 2001. Kruppel-like factor 4 (gut-enriched Kruppel-like factor) inhibits cell proliferation by blocking G1/S progression of the cell cycle. J Biol Chem 276:30423-30428. 10.1074/jbc.M101194200

Cheung TH, and Rando TA. 2013. Molecular regulation of stem cell quiescence. Nature reviews Molecular cell biology 14:329-340. 10.1038/nrm3591

Christofk HR, Vander Heiden MG, Harris MH, Ramanathan A, Gerszten RE, Wei R, Fleming MD, Schreiber SL, and Cantley LC. 2008. The M2 splice isoform of pyruvate kinase is important for cancer metabolism and tumour growth. Nature 452:230-233. 10.1038/nature06734

Chung SH, Onoda N, Ishikawa T, Ogisawa K, Takenaka C, Yano Y, Hato F, and Hirakawa K. 2002. Peroxisome Proliferator - activated Receptor Gamma Activation Induces Cell Cycle Arrest via the

Peer] reviewing PDF | (2018:11:32868:2:0:NEW 9 Apr 2019) 
436

437

438

439

440

441

442

443

444

445

446

447

448

449

450

451

452

453

454

455

456

457

458

459

460

461

462

463

464

465

466

467

468

469

470

471

472

473 p53 - independent Pathway in Human Anaplastic Thyroid Cancer Cells. Japanese Journal of Cancer Research 93:1358-1365. 10.1111/j.1349-7006.2002.tb01245.x

Clarke MF, Dick JE, Dirks PB, Eaves CJ, Jamieson CH, Jones DL, Visvader J, Weissman IL, and Wahl GM. 2006. Cancer stem cells - perspectives on current status and future directions: AACR Workshop on cancer stem cells. Cancer Research 66:9339-9344. 10.1158/0008-5472.CAN-06-3126.

Coller HA, Sang L, and Roberts JM. 2006. A new description of cellular quiescence. PLoS biology 4:e83. 10.1371/journal.pbio.0040083

Collins NL, Reginato MJ, Paulus JK, Sgroi DC, LaBaer J, and Brugge JS. 2005. G1/S cell cycle arrest provides anoikis resistance through Erk-mediated Bim suppression. Molecular and cellular biology 25:5282-5291. 10.1128/MCB.25.12.5282-5291.2005.

Cretella D, Saccani F, Quaini F, Frati C, Lagrasta C, Bonelli M, Caffarra C, Cavazzoni A, Fumarola C, and Galetti M. 2014. Trastuzumab emtansine is active on HER-2 overexpressing NSCLC cell lines and overcomes gefitinib resistance. Molecular cancer 13:143. 10.1186/1476-4598-13-143

Daubriac J, Fleury-Feith J, Kheuang L, Galipon J, Saint-Albin A, Renier A, Giovannini M, Galateau-Sallé F, and Jaurand M-C. 2009. Malignant pleural mesothelioma cells resist anoikis as quiescent pluricellular aggregates. Cell death and differentiation 16:1146-1155. 10.1038/cdd.2009.32

Davies MA, Koul D, Dhesi H, Berman R, McDonnell TJ, McConkey D, Yung WA, and Steck PA. 1999. Regulation of Akt/PKB activity, cellular growth, and apoptosis in prostate carcinoma cells by MMAC/PTEN. Cancer Research 59:2551-2556.

Davison CA, Durbin SM, Thau MR, Zellmer VR, Chapman SE, Diener J, Wathen C, Leevy WM, and Schafer ZT. 2013. Antioxidant enzymes mediate survival of breast cancer cells deprived of extracellular matrix. Cancer Research 73:3704-3715. 10.1158/0008-5472.CAN-12-2482

DiMeo TA, Anderson K, Phadke P, Fan C, Perou CM, Naber S, and Kuperwasser C. 2009. A novel lung metastasis signature links Wnt signaling with cancer cell self-renewal and epithelial-mesenchymal transition in basal-like breast cancer. Cancer Res 69:5364-5373. 10.1158/0008-5472.CAN-08-4135

Du X-L, Yang H, Liu S, Luo M, Hao J, Zhang Y, Lin D, Xu X, Cai Y, and Zhan Q. 2009. Calreticulin promotes cell motility and enhances resistance to anoikis through STAT3 - CTTN - Akt pathway in esophageal squamous cell carcinoma. Oncogene 28:3714-3722. 10.1038/onc.2009.237

El-Deiry WS, Harper JW, O'Connor PM, Velculescu VE, Canman CE, Jackman J, Pietenpol JA, Burrell M, Hill DE, and Wang Y. 1994. WAF1/CIP1 is induced in p53-mediated G1 arrest and apoptosis. Cancer Research 54:1169-1174. 10.1007/BF01525643.

Elkon R, Rashi-Elkeles S, Lerenthal Y, Linhart C, Tenne T, Amariglio N, Rechavi G, Shamir R, and Shiloh Y. 2005. Dissection of a DNA-damage-induced transcriptional network using a combination of microarrays, RNA interference and computational promoter analysis. Genome biology 6:R43. 10.1186/gb-2005-6-5-r43.

Feng MX, Ma MZ, Fu Y, Li J, Wang T, Xue F, Zhang JJ, Qin WX, Gu JR, and Zhang ZG. 2014. Elevated autocrine EDIL3 protects hepatocellular carcinoma from anoikis through RGD-mediated integrin activation. Molecular cancer 13:226. 10.1186/1476-4598-13-226.

PeerJ reviewing PDF | (2018:11:32868:2:0:NEW 9 Apr 2019) 
474 Fofaria NM, and Srivastava SK. 2014. Critical role of STAT3 in melanoma metastasis through anoikis

475

476

477

478

479

480

481

482

483

484

485

486

487

488

489

490

491

492

493

494

495

496

497

498

499

500

501

502

503

504

505

506

507

508

509

510

511

512 resistance. Oncotarget 5:7051-7064. 10.18632/oncotarget.2251

Fofaria NM, and Srivastava SK. 2015. STAT3 induces anoikis resistance, promotes cell invasion and metastatic potential in pancreatic cancer cells. Carcinogenesis 36:142-150. 10.1093/carcin/bgu233

Gartel AL, and Tyner AL. 2002. The role of the cyclin-dependent kinase inhibitor p21 in apoptosis. Mol Cancer Ther 1:639-649. 10.1097/00008390-200206000-00014.

Glauche I, Moore K, Thielecke L, Horn K, Loeffler M, and Roeder I. 2009. Stem cell proliferation and quiescence--two sides of the same coin. PLoS Comput Biol 5:e1000447. 10.1371/journal.pcbi.1000447

Hall J, Guo G, Wray J, Eyres I, Nichols J, Grotewold L, Morfopoulou S, Humphreys P, Mansfield W, Walker R, Tomlinson S, and Smith A. 2009. Oct4 and LIF/Stat3 additively induce Kruppel factors to sustain embryonic stem cell self-renewal. Cell Stem Cell 5:597-609. 10.1016/j.stem.2009.11.003

Hannafon BN, and Ding WQ. 2015. Cancer stem cells and exosome signaling. Stem Cell Investig 2:11. 10.3978/j.issn.2306-9759.2015.05.02

Hermani A, De Servi B, Medunjanin S, Tessier PA, and Mayer D. 2006. S100A8 and S100A9 activate MAP kinase and NF-kappaB signaling pathways and trigger translocation of RAGE in human prostate cancer cells. Exp Cell Res 312:184-197. 10.1016/j.yexcr.2005.10.013

Holland JD, Klaus A, Garratt AN, and Birchmeier W. 2013. Wnt signaling in stem and cancer stem cells. Current Opinion in Cell Biology 25:254-264. 10.1016/j.ceb.2013.01.004.

Huang DW, Sherman BT, and Lempicki RA. 2008. Systematic and integrative analysis of large gene lists using DAVID bioinformatics resources. Nature protocols 4:44-57. 10.1038/nprot.2008.211.

Huang DW, Sherman BT, and Lempicki RA. 2009. Bioinformatics enrichment tools: paths toward the comprehensive functional analysis of large gene lists. Nucleic Acids Research 37:1-13. 10.1093/nar/gkn923.

Issemann I, and Green S. 1990. Activation of a member of the steroid hormone receptor superfamily by peroxisome proliferators. Nature 347:645-650. 10.1038/347645a 0

Ji H, Greening DW, Barnes TW, Lim JW, Tauro BJ, Rai A, Xu R, Adda C, Mathivanan S, Zhao W, Xue Y, Xu T, Zhu HJ, and Simpson RJ. 2013. Proteome profiling of exosomes derived from human primary and metastatic colorectal cancer cells reveal differential expression of key metastatic factors and signal transduction components. Proteomics 13:1672-1686. 10.1002/pmic.201200562

Kim SH, and Singh SV. 2019. Role of Krüppel-like Factor 4-p21CIP1 Axis in Breast Cancer Stem-like Cell Inhibition by Benzyl Isothiocyanate. Cancer Prevention Research 12:125-134. 10.1158/19406207.CAPR-18-0393

Kim SM, Kwon OJ, Hong YK, Kim JH, Solca F, Ha SJ, Soo RA, Christensen JG, Lee JH, and Cho BC. 2012 a. Activation of IL-6R/JAK1/STAT3 signaling induces de novo resistance to irreversible EGFR inhibitors in non-small cell lung cancer with T790M resistance mutation. Mol Cancer Ther 11:22542264. 10.1158/1535-7163.MCT-12-0311

Kim YN, Koo KH, Sung JY, Yun UJ, and Kim H. 2012b. Anoikis resistance: an essential prerequisite for tumor metastasis. Int J Cell Biol 2012:306879. 10.1155/2012/306879 
513 Kobayashi S, Shimamura T, Monti S, Steidl U, Hetherington CJ, Lowell AM, Golub T, Meyerson M, Tenen

514

515

516

517

518

519

520

521

522

523

524

525

526

527

528

529

530

531

532

533

534

535

536

537

538

539

540

541

542

543

544

545

546

547

548

549

550

551

DG, and Shapiro GI. 2006. Transcriptional profiling identifies cyclin D1 as a critical downstream effector of mutant epidermal growth factor receptor signaling. Cancer Research 66:11389-11398. 10.1158/0008-5472.CAN-06-2318.

Kupferman ME, Patel V, Sriuranpong V, Amornphimoltham P, Jasser SA, Mandal M, Zhou G, Wang J, Coombes K, Multani A, Pathak S, Silvio Gutkind J, and Myers JN. 2007. Molecular analysis of anoikis resistance in oral cavity squamous cell carcinoma. Oral Oncol 43:440-454. 10.1016/j.oraloncology.2006.04.016

Lamb R, Ablett MP, Spence K, Landberg G, Sims AH, and Clarke RB. 2013. Wnt pathway activity in breast cancer sub-types and stem-like cells. PloS one 8:e67811. 10.1371/journal.pone.0067811

Li H-M, Yang J-G, Liu Z-J, Wang W-M, Yu Z-L, Ren J-G, Chen G, Zhang W, and Jia J. 2017. Blockage of glycolysis by targeting PFKFB3 suppresses tumor growth and metastasis in head and neck squamous cell carcinoma. Journal of Experimental \& Clinical Cancer Research 36:7. 10.1186/s13046-0160481- 1.

Liao Y, Chiang K, Shieh J, Huang C, Shen C, Huang W, and Chen B. 2017. Epidermal growth factor-induced ANGPTL4 enhances anoikis resistance and tumour metastasis in head and neck squamous cell carcinoma. Oncogene 36:2228-2242. 10.1038/onc.2016.371.

Masuda T, Wada K, Nakajima A, Okura M, Kudo C, Kadowaki T, Kogo M, and Kamisaki Y. 2005. Critical role of peroxisome proliferator-activated receptor gamma on anoikis and invasion of squamous cell carcinoma. Clin Cancer Res 11:4012-4021. 10.1158/1078-0432.CCR-05-0087

Miller WA, Wuertz BR, and Ondrey FG. 2018. PPAR $\gamma$-Mediated p21 Induction in Aerodigestive Preneoplastic Cell Lines. Annals of Otology, Rhinology \& Laryngology 127:677-686. 10.1177/0003489418787833.

Mootha VK, Lindgren CM, Eriksson KF, Subramanian A, Sihag S, Lehar J, Puigserver P, Carlsson E, Ridderstråle M, and Laurila E. 2003. PGC-1alpha-responsive genes involved in oxidative phosphorylation are coordinately downregulated in human diabetes. Nature Genetics 34:267-273. 10.1038/ng 1180 .

Pacholewska A. 2017. Loget"-a Uniform Differential Expression Unit to Replace" logFC" and" log2FC. Matters 3:e201706000011.

Perucca P, Cazzalini O, Madine M, Savio M, Laskey RA, Vannini V, Prosperi E, and Stivala LA. 2009. Loss of p21CDKN1A impairs entry to quiescence and activates a DNA damage response in normal fibroblasts induced to quiescence. Cell Cycle 8:105-114. 10.4161/cc.8.1.7507

Reginato MJ, Mills KR, Paulus JK, Lynch DK, Sgroi DC, Debnath J, Muthuswamy SK, and Brugge JS. 2003. Integrins and EGFR coordinately regulate the pro-apoptotic protein Bim to prevent anoikis. Nat Cell Biol 5:733-740. 10.1038/ncb1026

Riley T, Sontag E, Chen P, and Levine A. 2008. Transcriptional control of human p53-regulated genes. Nature reviews Molecular cell biology 9:402-412. 10.1038/nrm2395.

Rowland BD, and Peeper DS. 2006. KLF4, p21 and context-dependent opposing forces in cancer. Nat Rev Cancer 6:11-23. 10.1038/nrc1780

Peer) reviewing PDF | (2018:11:32868:2:0:NEW 9 Apr 2019) 
552

553

554

555

556

557

558

559

560

561

562

563

564

565

566

567

568

569

570

571

572

573

574

575

576

577

578

579

580

581

582

583

584

585

586

587

588

589

Sang L, Coller HA, and Roberts JM. 2008. Control of the reversibility of cellular quiescence by the transcriptional repressor HES1. Science 321:1095-1100. 10.1126/science.1155998

Schafer ZT, Grassian AR, Song L, Jiang Z, Gerhart-Hines Z, Irie HY, Gao S, Puigserver P, and Brugge JS. 2009. Antioxidant and oncogene rescue of metabolic defects caused by loss of matrix attachment. Nature 461:109-113. 10.1038/nature08268

Schoenfeld J, Lessan K, Johnson NA, Charnock-Jones DS, Evans A, Vourvouhaki E, Scott L, Stephens R, Freeman TC, Saidi SA, Tom B, Weston GC, Rogers P, Smith SK, and Print CG. 2004. Bioinformatic analysis of primary endothelial cell gene array data illustrated by the analysis of transcriptome changes in endothelial cells exposed to VEGF-A and PlGF. Angiogenesis 7:143-156. 10.1007/s10456004-1677-0

Sertznig P, Seifert M, Tilgen W, and Reichrath J. 2007. Present concepts and future outlook: function of peroxisome proliferator-activated receptors (PPARs) for pathogenesis, progression, and therapy of cancer. J Cell Physiol 212:1-12. 10.1002/jcp.20998

Sheldrick EL, Derecka K, Marshall E, Chin EC, Hodges L, Wathes DC, Abayasekara DR, and Flint AP. 2007. Peroxisome-proliferator-activated receptors and the control of levels of prostaglandin-endoperoxide synthase 2 by arachidonic acid in the bovine uterus. Biochem J 406:175-183. 10.1042/BJ20070089

Shen C-J, Chan S-H, Lee C-T, Huang W-C, Tsai J-P, and Chen B-K. 2017. Oleic acid-induced ANGPTL4 enhances head and neck squamous cell carcinoma anoikis resistance and metastasis via up-regulation of fibronectin. Cancer letters 386:110-122. 10.1016/j.canlet.2016.11.012.

Sher I, Adham SA, Petrik J, and Coomber BL. 2009. Autocrine VEGF-A/KDR loop protects epithelial ovarian carcinoma cells from anoikis. Int J Cancer 124:553-561. 10.1002/ijc.23963

Shield KD, Ferlay J, Jemal A, Sankaranarayanan R, Chaturvedi AK, Bray F, and Soerjomataram I. 2017. The global incidence of lip, oral cavity, and pharyngeal cancers by subsite in 2012. CA Cancer J Clin 67:51-64. 10.3322/caac.21384

Simpson CD, Anyiwe K, and Schimmer AD. 2008. Anoikis resistance and tumor metastasis. Cancer Lett 272:177-185. 10.1016/j.canlet.2008.05.029

Su Z, Yang Z, Xu Y, Chen Y, and Yu Q. 2015. Apoptosis, autophagy, necroptosis, and cancer metastasis. Molecular cancer 14:48. 10.1186/s12943-015-0321-5

Subramanian A, Tamayo P, Mootha VK, Mukherjee S, Ebert BL, Gillette MA, Paulovich A, Pomeroy SL, Golub TR, Lander ES, and Mesirov JP. 2005. Gene set enrichment analysis: a knowledge-based approach for interpreting genome-wide expression profiles. Proc Natl Acad Sci U S A 102:1554515550. 10.1073/pnas.0506580102

Suh Y, Amelio I, Guerrero Urbano T, and Tavassoli M. 2014. Clinical update on cancer: molecular oncology of head and neck cancer. Cell Death Dis 5:e1018. 10.1038/cddis.2013.548

Taddei M, Giannoni E, Fiaschi T, and Chiarugi P. 2012. Anoikis: an emerging hallmark in health and diseases. The Journal of pathology 226:380-393. 10.1002/path.3000.

Tang Z, Li C, Kang B, Gao G, Li C, and Zhang Z. 2017. GEPIA: a web server for cancer and normal gene expression profiling and interactive analyses. Nucleic Acids Res 45:W98-W102. 10.1093/nar/gkx247

Peer) reviewing PDF | (2018:11:32868:2:0:NEW 9 Apr 2019) 
590

591

592

593

594

595

596

597

598

599

600

601

602

603

604

605

606

607

608

609

610

611

612

613

614

615

616

617

618

619

620

621

622

623

624

625

626

627

628

Tarazona S, García-Alcalde F, Dopazo J, Ferrer A, and Conesa A. 2011. Differential expression in RNA-seq: a matter of depth. Genome research 21:2213-2223. 10.1101/gr.124321.111

Thompson EW, Newgreen DF, and Tarin D. 2005. Carcinoma invasion and metastasis: a role for epithelialmesenchymal transition? Cancer Res 65:5991-5995; discussion 5995. 10.1158/0008-5472.CAN-050616

Toyota M, Miyazaki Y, Kitamura S, Nagasawa Y, Kiyohara T, Shinomura Y, and Matsuzawa Y. 2002. Peroxisome proliferator-activated receptor $\gamma$ reduces the growth rate of pancreatic cancer cells through the reduction of cyclin D1. Life sciences 70:1565-1575. 10.1016/S0024-3205(01)01524-7

Vainio P, Gupta S, Ketola K, Mirtti T, Mpindi J-P, Kohonen P, Fey V, Perälä M, Smit F, and Verhaegh G. 2011. Arachidonic acid pathway members PLA2G7, HPGD, EPHX2, and CYP4F8 identified as putative novel therapeutic targets in prostate cancer. The American journal of pathology 178:525-536. 10.1016/j.ajpath.2010.10.002.

Viloriapetit A, Crombet T, Jothy S, Hicklin D, Bohlen P, Schlaeppi JM, Rak J, and Kerbel RS. 2001. Acquired Resistance to the Antitumor Effect of Epidermal Growth Factor Receptor-blocking Antibodies in Vivo: A Role for Altered Tumor Angiogenesis. Cancer Research 61:5090-5101.

Viswakarma N, Jia Y, Bai L, Vluggens A, Borensztajn J, Xu J, and Reddy JK. 2010. Coactivators in PPARRegulated Gene Expression. PPAR Res 2010. 10.1155/2010/250126

Vitolo MI, Weiss MB, Szmacinski M, Tahir K, Waldman T, Park BH, Martin SS, Weber DJ, and Bachman KE. 2009. Deletion of PTEN Promotes Tumorigenic Signaling, Resistance to Anoikis, and Altered Response to Chemotherapeutic Agents in Human Mammary Epithelial Cells. Cancer Research 69:8275-8283. 10.1158 / 0008-5472.CAN-09-1067.

Wei D, Kanai M, Huang S, and Xie K. 2006. Emerging role of KLF4 in human gastrointestinal cancer. Carcinogenesis 27:23-31. 10.1093/carcin/bgi243

Wreesmann VB, Shi W, Thaler HT, Poluri A, Kraus DH, Pfister D, Shaha AR, Shah JP, Rao PH, and Singh B. 2004. Identification of novel prognosticators of outcome in squamous cell carcinoma of the head and neck. J Clin Oncol 22:3965-3972. 10.1200/JCO.2004.01.094

Yadav A, Kumar B, Datta J, Teknos TN, and Kumar P. 2011. IL-6 promotes head and neck tumor metastasis by inducing epithelial - mesenchymal transition via the JAK-STAT3-SNAIL signaling pathway. Molecular Cancer Research 9:1658-1667. 10.1158/1541-7786.MCR-11-0271

Yan SR, Joseph RR, Rosen K, Reginato MJ, Jackson A, Allaire N, Brugge JS, Jobin C, and Stadnyk AW. 2005. Activation of NF- $\mathrm{K}$ B following detachment delays apoptosis in intestinal epithelial cells. Oncogene 24:6482-6491. 10.1038/sj.onc. 1208810

Yao G. 2014. Modelling mammalian cellular quiescence. Interface focus 4:20130074. 10.1098/rsfs.2013.0074

Yin C, Li H, Zhang B, Liu Y, Lu G, Lu S, Sun L, Qi Y, Li X, and Chen W. 2013. RAGE-binding S100A8/A9 promotes the migration and invasion of human breast cancer cells through actin polymerization and epithelial - mesenchymal transition. Breast Cancer Research \& Treatment 142:297-309. 10.1007 / s10549-013-2737-1.

Yong HY, and Moon A. 2007. Roles of calcium-binding proteins, S100A8 and S100A9, in invasive phenotype of human gastric cancer cells. Arch Pharm Res 30:75-81.

Peer] reviewing PDF | (2018:11:32868:2:0:NEW 9 Apr 2019) 
629 Zeng Q, Chen S, You Z, Yang F, Carey TE, Saims D, and Wang C-Y. 2002. Hepatocyte growth factor inhibits

630 anoikis in head and neck squamous cell carcinoma cells by activation of ERK and Akt signaling

631

632 independent of NF к B. Journal of Biological Chemistry 277:25203-25208. 10.1074 /

633 jbc.M201598200

634

Zhao D, Pan C, Sun J, Gilbert C, Drews-Elger K, Azzam D, Picon-Ruiz M, Kim M, Ullmer W, and El-Ashry

635 D. 2015. VEGF drives cancer-initiating stem cells through VEGFR-2/Stat3 signaling to upregulate Myc and Sox2. Oncogene 34:3107-3119. 10.1038/onc.2014.257.

636 


\section{Figure 1}

Cluster analysis of DEGs and RT-PCR confirmation.

Cluster Heatmap of DEGs (|Loget $\mid \geq 3, P \geq 0.9$ ) was generated by OmicShare tools (http://www.omicshare.com/tools/). (A) Cluster heatmap of the most significantly changed 360 DEGs (|Loget $\mid \geq 3$, probability $\geq 0.9$ ). The color of the squares in the heatmap reflects the $z$-score. The result of cluster analysis indicated that the samples had low heterogeneity and high repeatability. (B) To verify our RNA-seq results, we selected 21 genes, including 15 keratin-related genes and 6 randomly selected other genes, for examination by using RTPCR. GAPDH was selected as control. ALOX5AP has an independent control. All the detected 21 genes matched the results of RNA-Seq. It is revealed that high-throughput sequencing results are reliable. 

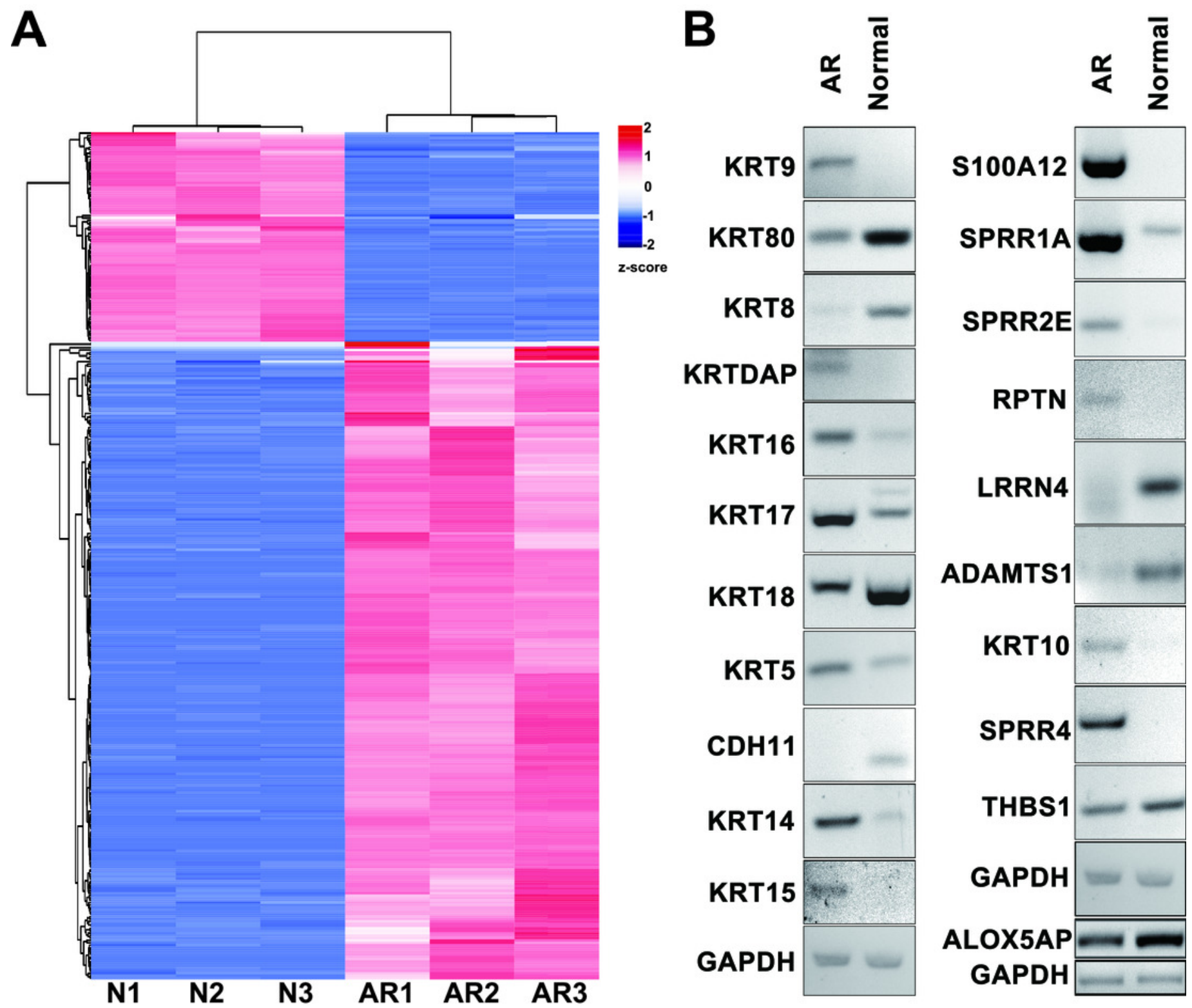
Figure 2 (on next page)

Gene ontology enrichment analysis of DEGs.

To facilitate the comprehensive analysis of DEGs, a total of 487 up-regulated genes and 335 down-regulated genes (|Loget $\mid \geq 2$, probability $\geq 0.9$ ) were analyzed using DAVID. (A) The top ten terms of biological process (BP), enriched in up- or down- regulated DEGs. (B) The top ten terms of molecular function (MF), enriched in up- or down- regulated DEGs. (C) The top ten terms of cellular component (CC), enriched in up- or down- regulated DEGs. p-value $\leq$ $0.05, F D R \leq 0.05$. 


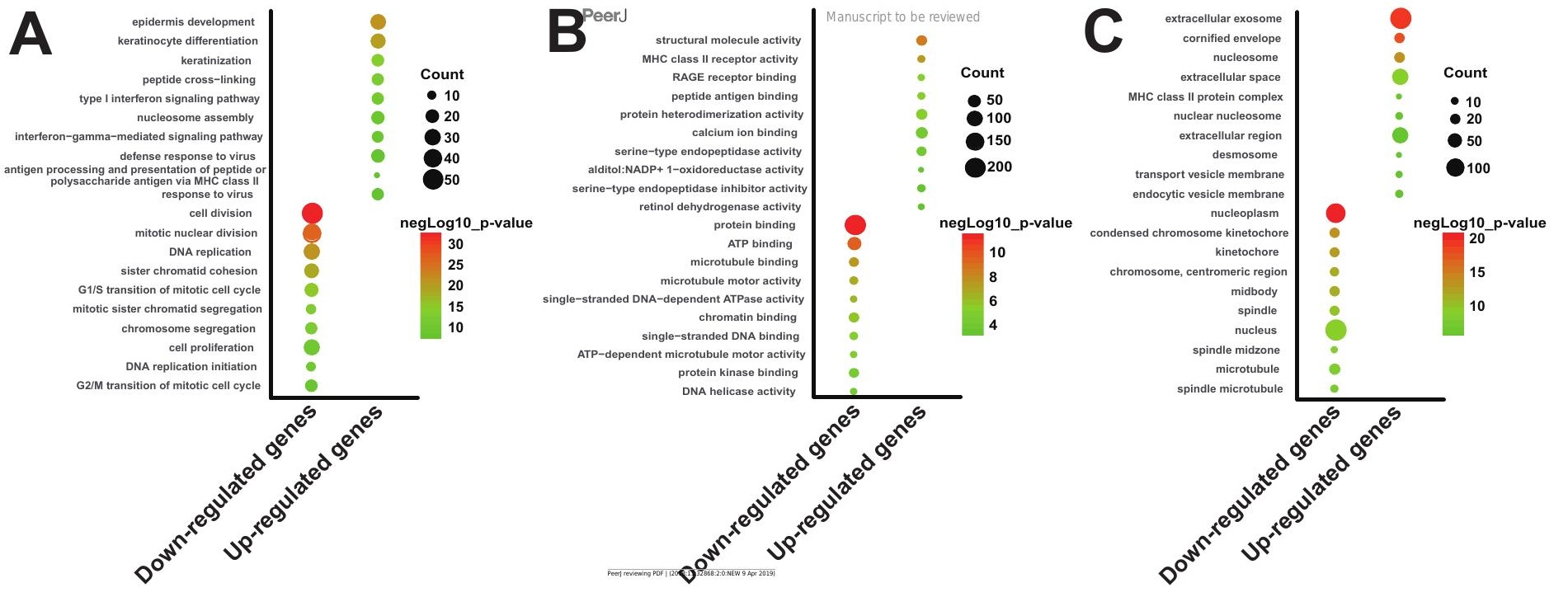


Figure 3 (on next page)

KEGG pathway analysis of DEGs using GSEA.

To further deliberate signal pathways of DEGs, the KEGG pathway that correlates with the

DEGs was studied using GSEA. (A) Activated pathway in CAL27 ${ }^{\text {AR }}$ cells, including drug metabolism cytochrome P450, PPAR signaling pathway, cell adhesion molecules (Cams), arachidonic metabolism, linoleic metabolism and Toll like receptor signaling pathway etc. (B) Suppressive pathway in CAL2 $7^{A R}$ cells, including DNA replication, mismatch repair, nucleotide excision repair and cell cycle pathways etc. 
KEGG_AUTOIMMUNE_THYROID_DISEASE KEGG_STEROID_HORMONE_BIOSYNTHESIS . KEGG_ALLOGRAFT_REJECTION

KEGG_METABOLISM_OF_XENOBIOTICS_BY_CYTOCHROME_P450. KEGG_RETINOL_METABOLISM

KEGG LINOLEIC ACID METABOLISM

KEGG_DRUG_METABOLISM_CYTOCHROME_P450 KEGG_GRAFT_VERSUS_HOST_DISEASE .

KEGG_TYPE_I_DIABETES_MELLITUS

KEGG_SYSTEMIC_LUPUS_ERYTHEMATOSUS

KEGG_ARACHIDONIC_ACID_METABOLISM

KEGG GLYCOSAMINOGLYCAN_DEGRADATION

KEGG_INTESTINAL_IMMUNE_NETWORK_FOR_IGA_PRODUCTION KEGG_ANTIGEN_PROCESSING_AND_PRESENTATION KEGG_ALDOSTERONE_REGULATED_SODIUM_REABSORPTION . KEGG_LEISHMANIA_INFECTION KEGG_RIG_I_LIKE_RECEPTOR_SIGNALING_PATHWAY KEGG_VIRAL_MYOCARDITIS KEGG_LYSOSOME

KEGG_PPAR_SIGNALING_PATHWAY KEGG_TOLL_LIKE_RECEPTOR_SIGNALING_PATHWAY KEGG_CELL_ADHESION_MOLECULES CAMS

\section{$\begin{array}{lllll}0.4 & 0.5 & 0.6 & 0.7 & 0.8\end{array}$ \\ Gene ratio}

FDR qvalue

0.03

0.02

0.01

0.00

SIZE

- 30

60

90

G_HOMO

KEGG_DNA_REPLICATION . LOGOUS_RECOMBINATION KEGG_MISMATCH_REPAIR

KEGG_RIBOSOME

KEGG_BASE_EXCISION_REPAIR KEGG_NUCLEOTIDE_EXCISION_REPAIR KEGG_CELL_CYCLE

KEGG_ONE_CARBON_POOL_BY_FOLATE.

KEGG_PROTEASOME

KEGG_RNA_POLYMERASE

KEGG_SPLICEOSOME

S_OF_UNSATURATED_FATTY_ACIDS

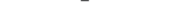

KEGG_PROTEIN_EXPORT
KEGG_OOCYTE_MEIOSIS

KEGG_PROGESTERONE_MEDIATED_OOCYTE_MATURATION
KEGG_CYSTEINE_AND_METHIONINE_METABOLISM KEGG_PURINE_METABOLISM

KEGG_AMINOACYL_TRNA_BIOSYNTHESIS KEGG_RNA_DEGRADATION KEGG_HYPERTROPHIC_CARDIOMYOPATHY_HCM . KEGG_FOCAL_ADHESION
FDR qvalue

0.04

0.03

0.02

0.01

0.00

SIZE

- 40

80

120 


\section{Figure 4 (on next page)}

Oncogenic signatures and CGP analysis of DEGs using GSEA.

To further deliberate the biological significance of DEGs, DEGs were analyzed using GSEA of Oncogenic signatures and CGP. (A) Up-regulated genes were enriched in the "VEGF_A_UP.V1_UP" term. (B) Upregulated genes were enriched in the "P53_DN.V2_UP" term. (C) Down-regulated genes were enriched in the "VEGF_A_UP.V1_DN" term. (D) Up-regulated genes were enriched in the "KOBAYASHI_EGFR SIGNALING_24HR_UP" term. (E) Up-regulated genes were enriched in the "YAN_ESCAPE_FROM_ANOIKIS "term. (F) Up-regulated genes were enriched in the "KOBAYASHI_EGFR_SIGNALING_24HR_DN" term. 
Enrichment plot: VEGF_A_UP.V1_UP

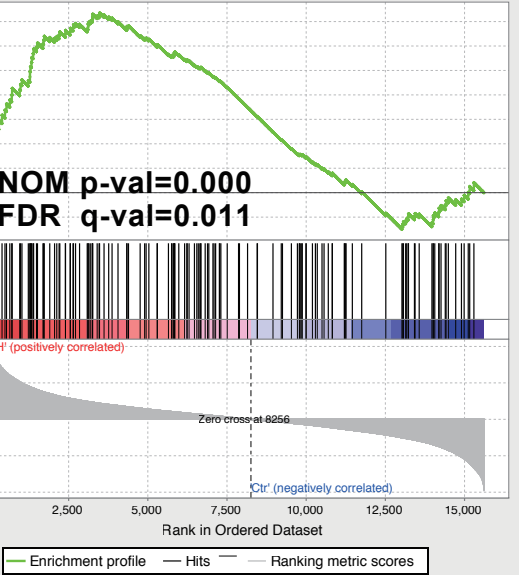

Enrichment plot:
KOBAYASHI_EGFR_SIGNALING_24HR_UP

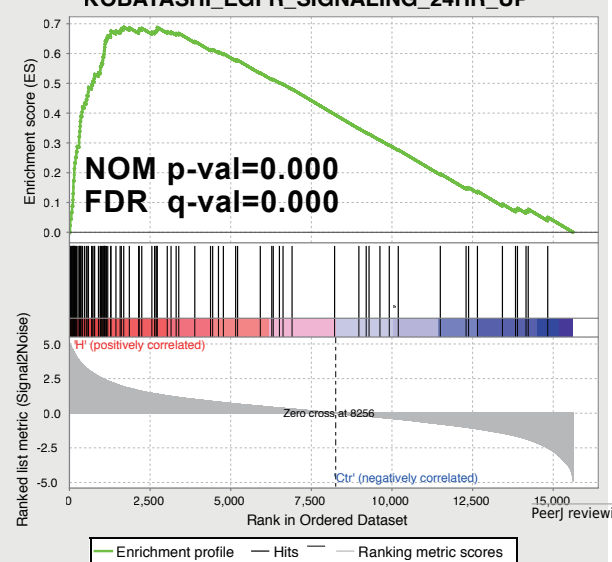

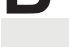

Enrichment plot: P53_DN.V2_UP
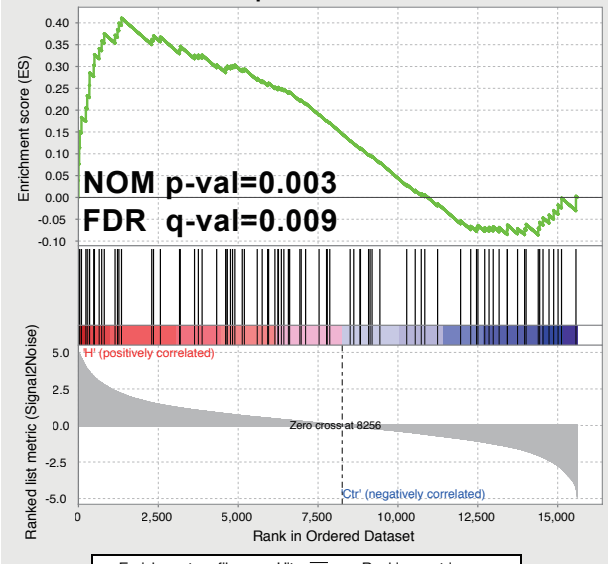

들

Enrichment plot: YAN_ESCAPE_FROM_ANOIKIS
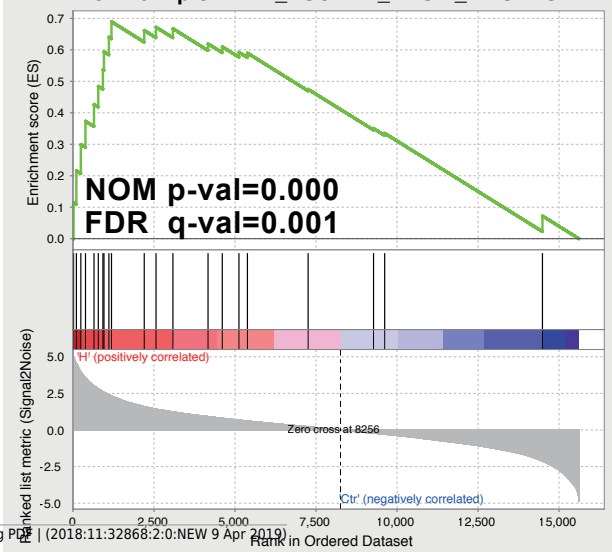

- Enrichment profile - Hits -- Ranking metric scores

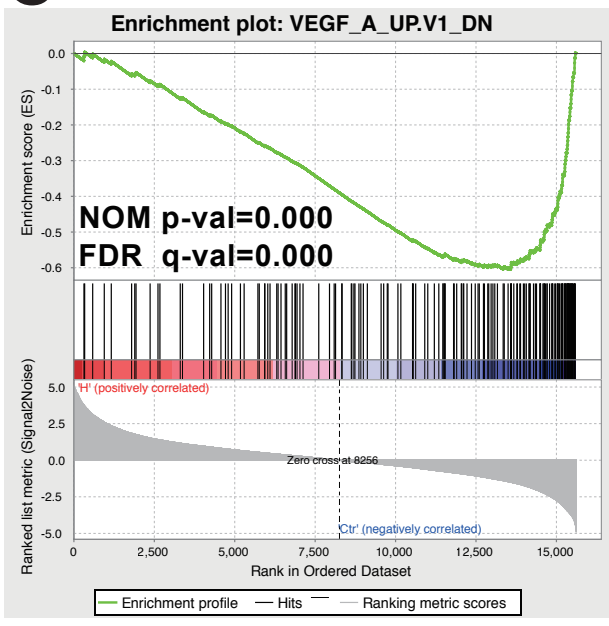

Enrichment plot:

KOBAYASHI_EGFR_SIGNALING_24HR_DN

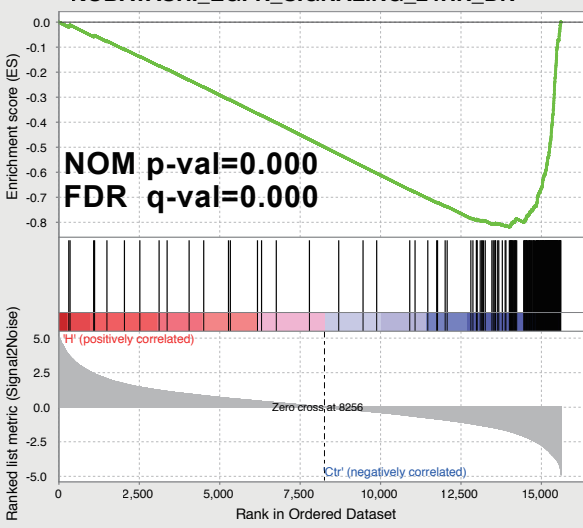

- Enrichment profile - Hits - - Ranking metric scores 


\section{Figure 5}

VEGFA-STAT3-KLF4-CDKN1A signal axis may be activated in CAL27 $7^{\text {AR }}$ cells

(A) RT-PCR showing that EGFR and IL-6 were down-regulated in CAL27 ${ }^{\text {AR }}$ cells. PDGFA and VEGFA were up-regulated in CAL27 ${ }^{\text {AR }}$ cells. (B) RT-PCR showing that CDKN1A and KLF4 were up-regulated in CAL27 ${ }^{\text {AR }}$ cells. The expression of TP53 was down-regulated in CAL27 ${ }^{\text {AR }}$ cells. (C) Western blotting analysis showing that the expression ofphospho-STAT3 (Tyr 705) proteincould only be detected in CAL2 $7^{\text {AR }}$ cells. Both CAL27 $7^{\text {AR }}$ and CAL27 cells could express phospho-STAT3 (Ser 727) protein. (D) The function analysis of down-regulated genes related to the term "VEGF_A_UP.V1_DN" using DAVID. The results showing that overexpression of VEGFA might negatively influence cell cycle. These cell cycle processes and eventsincluded DNA replication and G1/S transition. (E)-(I) Gene correlation analysis in HNSCC patients. (E) The correlation between VEGFA and KLF4 was positive. (F) The correlation between VEGFA and CDKN1A was positive. (G) The correlation between STAT3 and KLF4 was positive. (H) The correlation between STAT3 and CDKN1A was positive. (I) The correlation between KLF4 and CDKN1A was positive. 

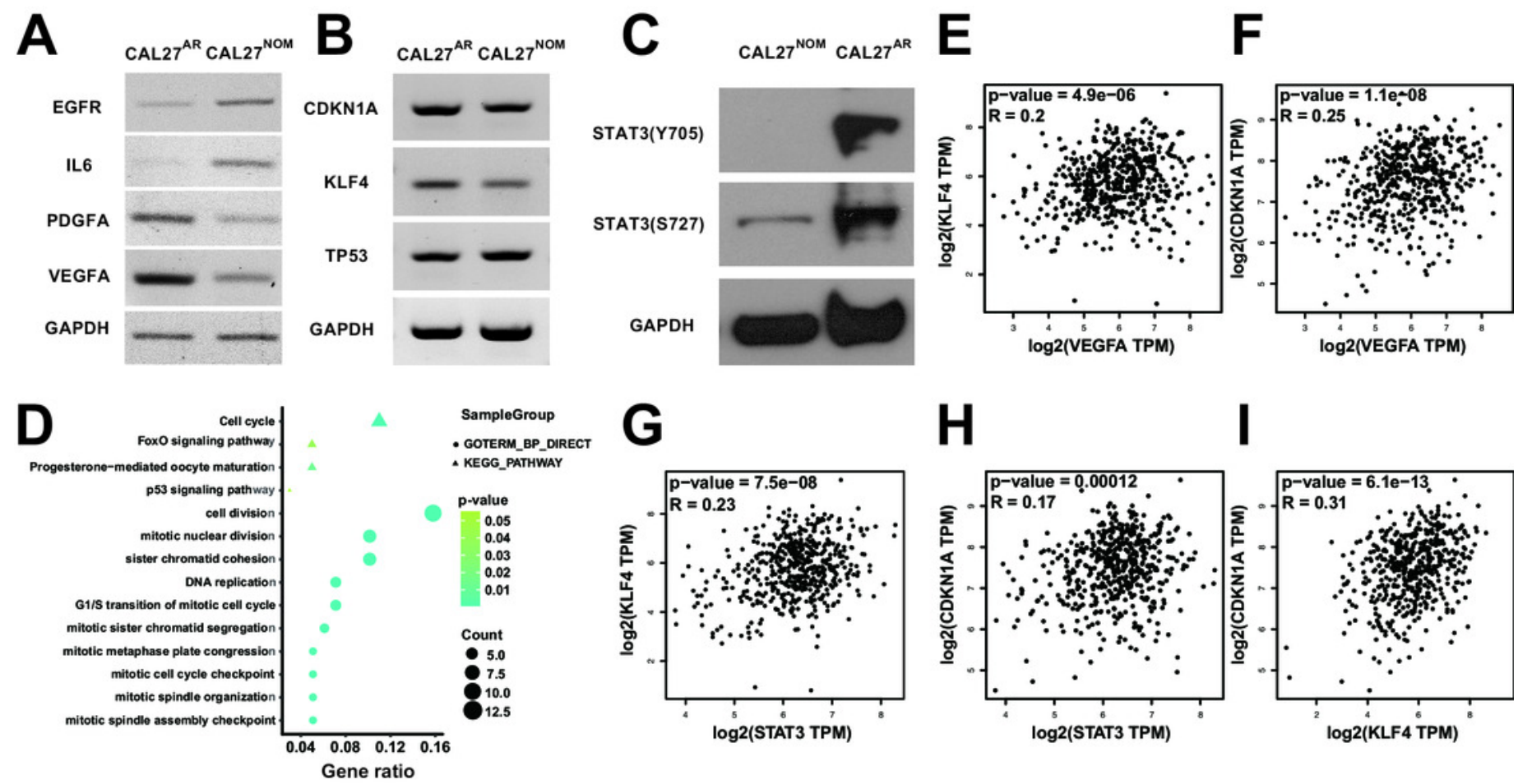
Figure 6 (on next page)

Cancer type that highly expressed VEGFA gene.

We studied the cancer type with high expression of VEGFA based on the TCGA database using GEPIA. (A)The expression of VEGFA in different tumor tissues. (B) The comparation of VEGFA expression among tissues of HNSCC, KIRC, OV, GSM and corresponding normal tissues. The results revealed that VEGFA also was expressed highly in kidney renal clear cell carcinoma (KIRC), ovarian serous cystadenocarcinoma (OV) and the glioblastoma (GBM), in addition to HNSCC. 


\section{Figure 7 (on next page)}

Gene correlation analysis of KIRC, OV and GBM.

The gene correlations among VEGFA, STAT3, KLF4 and CDKN1A in KIRC ,OV and GSM were analyzed using GEPIA. (A-E) The result of gene correlation analysis of KIRC. (F-J) The result of gene correlation analysis of OV. (K-O) The result of gene correlation analysis of GBM. The results showed that there was a positive correlation between KLF4 and CDKN1A in KIRC, OV and GBM. STAT3 had a positive correlation with KLF4 and CDKN1A, respectively, in KIRC, OV and GBM. In addition, VEGFA also has a positive correlation with KLF4 and CDKN1A, respectively in KIRC and OV ( $p$-value $\leq 0.05$ ). However, there is no statistically significant correlation between VEGFA and KLF4 in GBM. 
